\title{
Multiple Unilateral Vestibular Schwannomas: Segmental NF2 or Sporadic Occurrence?
}

\author{
Matthew L. Carlson ${ }^{1,2}$ Jamie J. Van Gompel ${ }^{1,2}$ \\ ${ }^{1}$ Department of Otolaryngology-Head and Neck Surgery, Mayo Clinic, \\ Rochester, Minnesota, United States \\ ${ }^{2}$ Department of Neurologic Surgery, Mayo Clinic, Rochester, \\ Minnesota, United States \\ J Neurol Surg Rep 2016;77:e106-e108.
}

\begin{abstract}
Address for correspondence Matthew L. Carlson, MD, Department of Otolaryngology-Head and Neck Surgery, Mayo Clinic, 200 First St. SW, Rochester, MN 55905, United States (e-mail: carlson.matthew@mayo.edu).
\end{abstract}

\begin{abstract}
Keywords

- vestibular schwannoma

- internal auditory canal

- magnetic resonance imaging

- neurofibromatosis type 2

Objective To report a case of a patient presenting with two separate unilateral vestibular schwannomas (VSs) without other stigmata of neurofibromatosis type 2 (NF2).

Study Design This article discusses a case report and review of the literature.

Setting Tertiary academic referral center.

Participants A 41-year-old female was referred for evaluation of a left-sided 1.8-cm cerebellopontine angle tumor centered on the porus acusticus and a separate ipsilateral 3-mm intracanalicular tumor appearing to arise from the superior vestibular nerve. The patient denied a family history of NF2. Neurotologic examination was unremarkable and close review of magnetic resonance imaging did not find any other stigmata of NF2. Results The patient underwent left-sided retrosigmoid craniotomy with gross total resection of both tumors. Final pathology confirmed benign schwannoma. The INI1/SMARCB1 staining pattern did not suggest NF2 or schwannomatosis.

Conclusions This is only the third report of a case with multiple unilateral VSs occurring in a patient without other features of NF2. Herein, the authors review the two other reports and discuss potential mechanisms for this rare phenomenon.
\end{abstract}

\section{Introduction}

The majority of vestibular schwannomas (VSs) are unilateral, while less than $5 \%$ are associated with neurofibromatosis type 2 (NF2), a high-penetrance autosomal dominant disease hallmarked by the development of bilateral VS. Approximately half of newly diagnosed cases of NF2 have no known family history of disease and arise from a de novo mutation in the NF2 gene occurring during the postzygotic stages of embryogenesis. Phenotypic expression within the NF2 population is variable, with some patients manifesting a mild disease variant with small bilateral VS diagnosed at an old age, while others presenting at a young age with innumerable intracranial and spinal tumors resulting in severe debilitation and premature death. Patients with moderate or severe phenotypes may

received

February 14, 2016 accepted after revision

May 9, 2016

published online

xxxx
DOI http://dx.doi.org/
10.1055/s-0036-1584603 ISSN 2193-6358. develop collision tumors or multiple separate tumors along the course of the eighth cranial nerve. ${ }^{1}$ This phenomenon is generally considered to be unique to the NF2 population. To the authors' knowledge, only two cases of unilateral multifocal VS have been reported in the English literature. ${ }^{2,3}$ Herein, the authors present a case of a medial VS isolated to the cerebellopontine angle (CPA) and a discretely separate small intracanalicular VS in the same ear.

\section{Case Presentation}

An otherwise healthy 41-year-old female was referred for treatment of a recently diagnosed left-sided $1.8-\mathrm{cm} \mathrm{CPA}$ tumor centered on the internal auditory canal (IAC) and a separate ipsilateral 3-mm intracanalicular tumor appearing
License terms

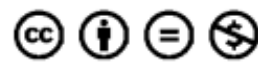

(c) 2016 Georg Thieme Verlag KG Stuttgart · New York 


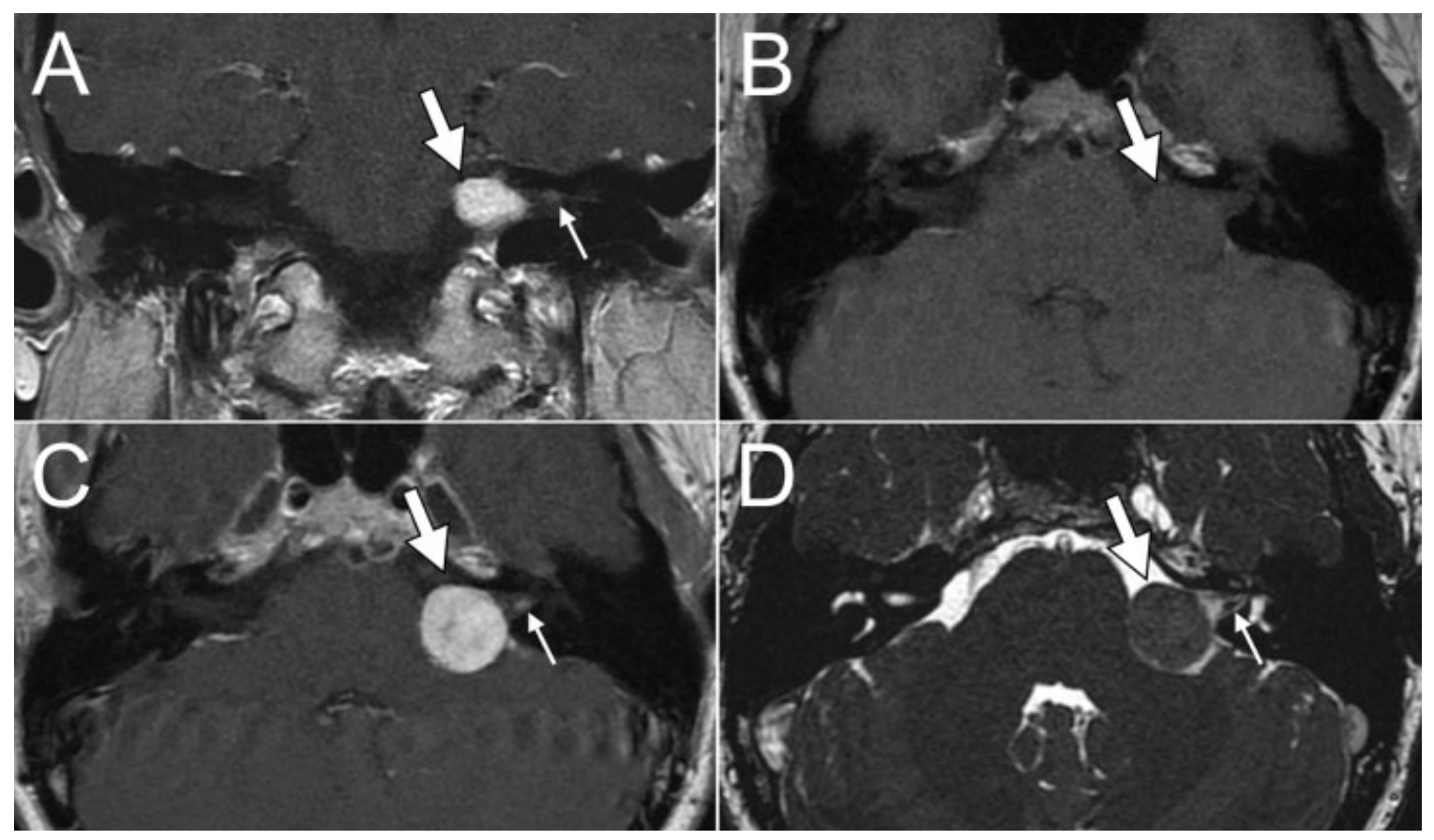

Fig.1 (A) Coronal and (C) axial T1-weighted magnetic resonance imaging (MRI) with contrast demonstrating a 1.8-cm cerebellopontine angle lesion (thick white arrow) centered on the internal auditory canal, without meatal extension. A separate 3-mm tumor (thin white arrow) can be seen in the distal internal auditory canal, distinctly separate from the cisternal tumor. (B) Axial precontrast T1-weighted MRI demonstrates a leftsided isointense cerebellopontine angle lesion. Without contrast administration, the meatal tumor cannot be identified. (D) Axial heavy T2weighted MRI (constructive interference steady state) further characterizing two separate tumors involving the left cerebellopontine angle and internal auditory canal consistent with multiple unilateral vestibular schwannomas.

to arise from the superior vestibular nerve (-Fig. 1). The patient reported a history of several separate episodes of sudden sensorineural hearing loss that were steroid responsive, the first occurring 3 years prior. A magnetic resonance imaging (MRI) was ultimately obtained after her third episode of sudden hearing loss, establishing the diagnosis of VS. The patient denied a family history of NF2. Neurotologic examination was unremarkable and close review of MRI did not find any other stigmata of NF2. Audiometric testing revealed AAO-HNS (American Academy of Otolaryngology-Head and Neck Surgery) class A hearing bilaterally, with asymmetrical pure tone thresholds and 92\% word recognition in the left ear. Genetic testing for NF2 was discussed with the patient but not pursued. After reviewing management options, the patient was brought to the operating room and underwent a left retrosigmoid craniotomy with resection of the larger cisternal tumor and the separate smaller intracanalicular tumor (-Fig. 2). Final histologic analysis revealed benign schwannoma for both separate specimens. Postoperatively, the patient had normal facial nerve function and an uneventful recovery. A 3-month postoperative MRI confirmed gross total resection and audiometry demonstrated class $D$ hearing with

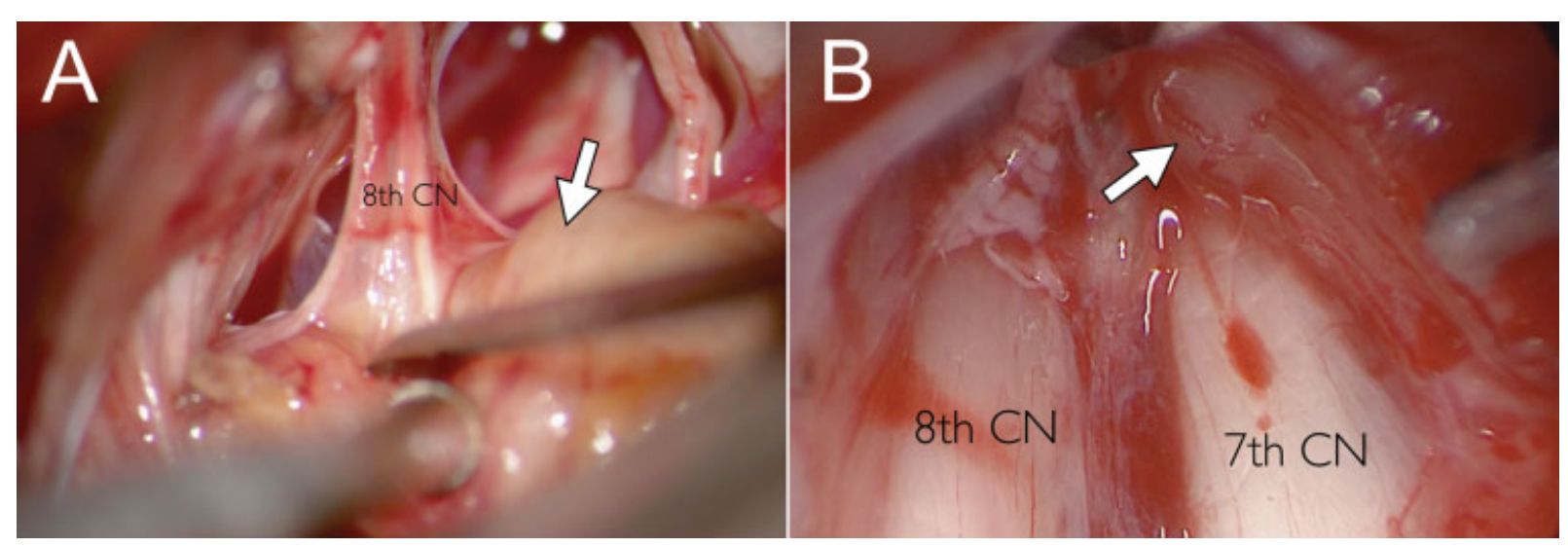

Fig. 2 (A) Left-sided retrosigmoid craniotomy with resection of the larger cisternal vestibular schwannoma (white arrow). (B) Endoscopic view of the separate smaller intracanalicular vestibular schwannoma (white arrow). 
a $47-\mathrm{dB}$ pure tone average and a $30 \%$ word recognition score. The INI1/SMARCB1 staining pattern did not suggest NF2 or schwannomatosis. ${ }^{4}$

\section{Discussion}

To the authors' knowledge, this is only the third report of a case with multiple unilateral VSs occurring in a patient without other features of NF2. ${ }^{2,3}$ In the following, we review the characteristic imaging features of VS and the radiologic differential diagnosis, summarize the two prior reports of multifocal unilateral VS, and finally offer several explanations for this rare and baffling phenomenon.

High-resolution MRI with gadolinium allows for accurate diagnosis of VS, even less than $2 \mathrm{~mm}$ in size. VS classically exhibit hypo- to isointense signal on precontrast T1-weighted imaging, iso- to hyperintense signal on T2-weighted sequences, and avid enhancement with contrast administration. VSs involving the CPA are classically centered on the IAC and usually exhibit meatal extension. In contrast, meningiomas are generally eccentric to the porus acusticus, exhibit a broad base with dural tails, and often display hyperostosis of underlying bone with intratumoral calcifications seen on computed tomography. Epidermoids of the CPA are usually isointense to cerebrospinal fluid on T1- and T2-weighted MRI and do not enhance with contrast. They are most easily distinguished from other CPA lesions using diffusion-weighted imaging, uniquely revealing restricted diffusion. Lastly, lipomas are distinctively hyperintense on precontrast T1- and hypointense on T2-weighted sequences and readily suppress with fat saturation sequences. Additionally, epidermoids and lipomas distinguish themselves from VS and meningioma by their propensity to engulf coursing nerves and vessels rather than pushing them to the tumor periphery and are more apt to acquiesce to the contour of the cistern before causing significant brainstem compression.

The first report of a unilateral double VS was by Kennedy et al in $2005 .^{2}$ In this case, a subtle facial nerve paresis prompted an MRI study leading to the diagnosis of unilateral multifocal VSs-one medially based cisternal tumor and a second small intracanalicular VS. Additional clinical details, including patient age and tumor dimensions, were not specified. Subsequently, Barbara et $\mathrm{al}^{3}$ reported the case of a 47-year-old man who was discovered to have two unilateral VSs after developing asymmetrical hearing loss. In this case, the patient did not have any family history of disease, and genetic testing of blood and normal skin was negative for NF2 mutation. Notably, however, the patient also had numerous uncharacterized "fibromalike masses" of the scalp. In the currently presented case, the patient denied a family history of NF2, and INI1/SMARCB1 staining suggested that the tumors were not associated with NF2 or schwannomatosis.

There are several plausible explanations for the development of multiple unilateral VS. First, it is conceivable that this condition resulted from mosaic NF2, where a mutation occurred sometime after conception, leading to two separate cell lineages. In this scenario, if a de novo mutation of the NF2 gene develops late in embryogenesis, segmental NF2 may progress explaining unilateral and often localized manifestations of NF2. However, in such cases, patients may never truly fulfill Manchester criteria for NF2, and lymphocyte genetic testing is frequently negative since the mutation may never affect leukocyte lineages. This theory may explain the finding of Barbara et $\mathrm{al}^{3}$ where a patient had multifocal unilateral VSs and multiple subcutaneous "fibromas" of the scalp, but negative blood genetic testing. It is also important to realize that among patients without a family history of disease, a mutation can only be positively detected in approximately $80 \%$ of patients and therefore a negative test does not guarantee freedom from future NF2 diagnosis.

It is also possible that multifocal unilateral VS is the heralding presentation in a minority of patients who will eventually fulfill diagnostic criteria for NF2. However, it is well established that the great majority of patients with NF2 initially present with bilateral tumors, and sequential VS development after the age of 40 is exceptionally rare. ${ }^{5}$ Lastly, it is conceivable that the occurrence of multiple unilateral VS is more common than we realize and was only diagnosed in these select cases because the medial tumor did not venture into the IAC and join with the intracanalicular tumor. That is, perhaps a small fraction of sporadic VS are actually a confluent mass comprising several adjacent tumors. This hypothesis is further supported by histologic studies of sporadic tumors demonstrating multicentric involvement of the vestibular nerve. 6

\section{Institutional Review Board Approval} 15-001365

\section{Funding}

No funding was required for this report.

Conflict of Interest

There are no relevant conflicts of interest to disclose.

\section{References}

1 Stivaros SM, Stemmer-Rachamimov AO, Alston R, et al. Multiple synchronous sites of origin of vestibular schwannomas in neurofibromatosis type 2. J Med Genet 2015;52(8):557-562

2 Kennedy RJ, Salzman KL, Shelton C. Unilateral double vestibular schwannoma. Otol Neurotol 2005;26(6):1241-1242

3 Barbara M, Ronchetti F, Manni V, Monini S. Double localization of a unilateral sporadic vestibular schwannoma. Acta Otorhinolaryngol Ital 2008;28(1):34-37

4 Patil S, Perry A, Maccollin M, et al. Immunohistochemical analysis supports a role for INI1/SMARCB1 in hereditary forms of schwannomas, but not in solitary, sporadic schwannomas. Brain Pathol 2008;18(4):517-519

5 Evans DG, Lye R, Neary W, et al. Probability of bilateral disease in people presenting with a unilateral vestibular schwannoma. J Neurol Neurosurg Psychiatry 1999;66(6):764-767

6 Luetje CM, Whittaker CK, Callaway LA, Veraga G. Histological acoustic tumor involvement of the VIIth nerve and multicentric origin in the VIIIth nerve. Laryngoscope 1983;93(9):1133-1139 